\title{
ÉTAT ET SCIENCES SOCIALES
}

States, social knowledge, and the origins of modern social policies. Éd. Dietrich Rueschemeyer et Theda Skocpol. Princeton/New York, Princeton University Press/Russell Sage Foundation, 1996. 15,5 × 23,5, 322 p., index.

Le recueil de Dietrich Rueschemeyer et Theda Skocpol prolonge la problématique d'un ouvrage antérieur intitulé Bringing the state back in (1985). Ce volume collectif, édité par les mêmes chercheurs accompagnés de Peter Evans, proposait un programme de recherches s'opposant à la fois à la vision instrumentaliste de l'État, qui avait été dominante dans les courants fonctionnalistes et marxistes, et au néolibéralisme émergent, qui ignorait les fonctions de l'État ou les réduisait au parasitisme économique du rent-seeking. Contre ces réductionnismes, la démarche alternative est fondée sur l'analyse de l'autonomie relative et l'efficacité propre des institutions étatiques. Pour tenir compte de la diversité de l'État moderne, une perspective historico-comparative s'est imposée, qui a permis d'étudier des trajectoires nationales parfois divergentes et ses modalités d'encastrement social. À travers l'analyse des variations, de nouvelles théories sociologiques se sont construites pour rendre compte à la fois des variants et des invariants.

Le livre de Theda Skocpol, States and social revolutions (1979), avait joué un rôle en quelque sorte pionnier. La sociologue d'Harvard a montré que les révolutions politiques de l'époque moderne s'expliquent essentiellement par une crise, notamment fiscale, de l'État (et non pas, par exemple, par la lutte des classes engendrée par l'exploitation économique des paysans). Son travail a été suivi par une série de travaux importants (Randall Collins, Peter Evans, Peter Hall, Michael Mann, Charles Tilly), faisant de la sociologie historique de l'État un domaine de recherche particulièrement vivant aux États-Unis.

Une question particulière qui s'est posée au cours des années quatrevingt dans ce cadre de recherches comparatives concernait le poids relatif Revue de synthèse : $4^{\mathrm{e}} \mathrm{S} . \mathrm{n}^{\text {os }} 3$-4, juil.-déc. 2000, p. 479-533. 
de la connaissance sociale et des experts intellectuels. Selon Skocpol et Rueschemeyer les analyses traditionnelles de la politique sociale ont presque toutes négligé le travail proprement cognitif dans l'émergence d'une politique sociale. Celles-ci ont ainsi laissé de côté la fonction majeure des groupes d'experts dans la redéfinition progressive des tâches étatiques, redéfinition qui fut à l'origine de l'État-providence. Poser cette question a produit une rencontre avec des spécialistes en histoire des sciences sociales, qui a donné lieu à plusieurs ouvrages collectifs. Social science and modern States (1991), édité par Peter Wagner, Carol Weiss, Björn Wittrock et Hellmut Wollmann, est une exploration générale de la question, centrée notamment sur le développement des policy sciences dans des contextes nationaux différents. The Political Power of economic ideas. Keynesianism across nations (1989), édité par Peter Hall, concerne plus particulièrement la restructuration des rapports entre experts économiques et pouvoir politique après la crise de 1929. Le recueil plus récent de Rueschemeyer et Skocpol porte plus largement sur les interactions entre science sociale et politique sociale entre 1870 et 1930 .

Le point de départ du recueil est le fait qu'au cours du $\mathrm{XIX}^{\mathrm{e}}$ siècle plusieurs problèmes nouveaux se sont posés aux élites nationales : celui de la croissance des inégalités produites par l'industrialisation, celui d'un élargissement du droit de vote, et, après 1870 , celui d'une compétition internationale plus intense avec un déclin économique relatif de l'Angleterre et la montée de l'Allemagne unifiée. Dans la perception de ces problèmes et dans les réponses politiques proposées, des groupes d'experts ont joué un rôle capital. Ne représentant ni la classe ouvrière et ses organisations, ni les industriels ou les propriétaires, ces policy intellectuals disposaient d'un savoir et d'une compétence spécifique tout en ayant des liens stratégiques avec les élites politiques et bureaucratiques. Cette configuration d'acteurs et d'institutions se trouve au cœur des trois parties du livre.

La première partie rassemble des études centrées sur l'évolution de la connaissance sociale et son contexte de production; la deuxième partie porte sur les rapports entre les groupes d'experts et l'État; la troisième concerne l'usage des connaissances sociales au sein des institutions étatiques. Les chapitres sont pour la plupart des études de cas, analysant une question précise dans une perspective comparative, c'est-à-dire dans au moins deux contextes nationaux. Des chapitres portent ainsi sur les sujets suivants: les accidents industriels en Allemagne et en France (Anson Rabinbach), les associations de sciences sociales allemandes et anglaises (Rueschemeyer et Van Rossum), le chômage et la recherche sociale en Angleterre et aux États-Unis (Libby Schweber), la politique du bien-être des enfants au Canada et aux États-Unis (John Stutton), la sécurité sociale dans les pays scandinaves (Stein Kuhnle), le rapport entre savoir social et État en Angleterre et au Japon (Sheldon Garon). 
Presque tous les chapitres essaient de ne pas limiter l'analyse à leur objet spécifique mais de poser des problèmes plus généraux. Le chapitre de Rueschemeyer et Van Rossum, par exemple, n'est pas seulement une comparaison détaillée du Verein für Sozialpolitik et de la Fabian Society mais touche à beaucoup d'autres aspects du fonctionnement de l'État en Allemagne et en Angleterre. Dans sa comparaison entre la recherche sociale anglaise et américaine, Libby Schweber jette une lumière nouvelle sur la question de savoir pourquoi la sociologie a réussi à s'établir si rapidement dans les universités américaines alors qu'elle ne s'est instituée en Angleterre que bien après la Seconde Guerre mondiale.

Outre l'introduction et la conclusion des éditeurs, deux chapitres présentent un cadre général permettant de mieux situer les différentes études de cas. Ira Katznelson discute la constitution, depuis le milieu du XIX ${ }^{\mathrm{e}}$ siècle, de ce qu'il appelle un "nouveau libéralisme». Ce libéralisme dit social, en rupture avec le libéralisme manchesterien d'un capitalisme sans frein et d'une politique de laissez faire, est intimement lié à l'émergence de la « science sociale » telle qu'elle est incarnée par des associations comme la Social Science Association en Angleterre, l'American Social Science Association ou le Verein für Sozialpolitik. Ce courant du libéralisme social a connu deux transformations majeures. D'abord la division progressive d'une science sociale relativement unifiée en disciplines distinctes, chacune avec ses propres chaires, revues et sociétés savantes. À cette structure de plus en plus disciplinaire s'ajoute ensuite un clivage plus net entre savants universitaires et amateurs éclairés. Le chapitre de Björn Wittrock et Peter Wagner présente un cadre plus général pour comprendre les variations nationales. Centrale dans leur analyse est l'opposition entre des sociétés ayant un État centralisé relativement fort et celles qui n'en ont pas. La distinction recouvre en gros celle entre les pays européens continentaux et les pays anglo-saxons. Plusieurs conséquences sont discutées, y compris celles qui concernent la formation des universités modernes et les structures institutionnelles des sciences sociales qui en découlent.

Contenant d'excellentes études de cas, ce recueil est exemplaire surtout pour sa démarche comparative. Des travaux rigoureusement comparatifs comme celui-ci existent encore à peine dans le domaine de l'histoire des sciences sociales. 
Robert CASTEL, Métamorphose de la question sociale. Une chronique du salariat. Paris, Fayard, 1995. $14 \times 24,488$ p., index (L'Espace du politique).

William Julius WILsON, When work disappears. The world of the new urban poor.

New York, Alfred A. Knopf, 1996. $17 \times 24,5,322$ p.

Pourquoi rendre compte ensemble de ces deux ouvrages? Tous deux publiés en 1996 par des sociologues éminents dans leurs pays respectifs, When work disappears et Métamorphose de la question sociale traitent le même sujet: les problèmes sociaux posés par le chômage. Ils entendent faire date tant dans l'univers de la recherche que dans l'espace politicomédiatique. Tous deux reposent sur une reformulation des termes du débat politique, administratif et scientifique et sont semblablement orientés vers l'action pratique et politique. Il paraissait donc intéressant de provoquer leur rencontre pour faire apparaître leurs convergences d'analyse, au-delà des importantes différences liées aux contextes nationaux.

Le projet de Robert Castel est de comprendre «cette nouvelle donne contemporaine: la présence, apparemment de plus en plus insistante, d'individus placés comme en situation de flottaison dans la structure sociale ». À cette fin, il faut respecter deux principes. D'abord, la situation de ces populations de «surnuméraires » ne trouve son explication que dans le rapport qu'elles entretiennent avec l'ensemble de la structure sociale. Ensuite, la crise contemporaine doit être comprise comme une crise non pas du travail en général mais de sa forme actuelle : le travail salarié. L'histoire montre que seule la relation salariée a permis de sortir de la vulnérabilité de masse, qui était le lot commun jusqu'au début du $\mathrm{xx}^{\mathrm{e}}$ siècle. Les premiers chapitres sont consacrés aux problèmes du vagabondage et mettent en évidence la lente et paradoxale émergence de la condition salariale qui représentait l'indignité absolue dans le bas Moyen Âge et devient le statut de presque $90 \%$ des salariés aujourd'hui. Les cinquième et sixième chapitres exposent comment la relation salariale se stabilise en même temps que (parce que) s'invente la notion de «propriété sociale», compromis presque miraculeux qui résout le vieil et insoluble dilemme entre l'assurance par la propriété et l'assurance par le travail. Le salaire n'est plus seulement la rétribution du travail par un employeur privé; il comporte aussi une part de cotisations sociales qui constituent une rente pour les situations de hors travail. Ainsi, cette propriété sociale, liée au travail, libère tant de l'hégémonie du travail que de celle du patrimoine. Cette relation salariale garantie par l'État est donc non seulement compatible avec l'organisation économique capitaliste mais elle la parachève en rendant possible une 
« association inédite de la sécurité et de la mobilité ». C'est pour cette raison que Castel récuse au passage l'expression d'État-providence et lui préfère celle d'État social : loin de donner sans contrepartie, l'État garantit la viabilité d'une organisation économique et sociale mise en péril par l'éclatement des réseaux de sociabilité primaire. On voit que l'enjeu de cette analyse est tout à fait contemporain. Dans le huitième et dernier chapitre, Castel s'élève avec force contre les contempteurs de l'intervention étatique et affirme : «[...] le tout économique n'a jamais fondé un ordre social; dans une société complexe, la solidarité n'est plus un donné mais un construit; la propriété sociale est à la fois compatible avec le patrimoine privé et nécessaire pour l'inscrire dans des stratégies collectives; le salaire, pour échapper à son indignité séculaire, ne peut se réduire à la rémunération d'une tâche. » Castel prend ensuite parti plus précisément encore et appelle de ses vœux un partage du travail encadré par l'intervention de l'État, seul moyen selon lui de sauvegarder la société salariale menacée aujourd'hui par la concurrence internationale et les formidables progrès de la productivité industrielle.

L'ouvrage de William Julius Wilson se présente comme l'analyse de trois enquêtes précises, menées à Chicago entre 1987 et 1989, mais ne s'interdit pas de convoquer un matériau plus large; il veut également rendre compte de manière générale de l'appauvrissement des ghettos noirs urbains et des classes moyennes américaines. Les deux premiers chapitres affirment que la polarisation de l'attention administrative, politique et scientifique autour des problèmes de la pauvreté occultent l'essentiel : la centralité du problème de la disparition de l'emploi. Les trois chapitres suivants mettent en évidence le cercle vicieux que forment ensemble la ghettoïsation de certains quartiers urbains noirs et la disparition du travail peu qualifié. Enfin, dans les trois derniers chapitres, Wilson propose un véritable programme d'intervention étatique autour de cinq axes : création d'emplois par l'État, développement de l'impôt négatif, élargissement de l'enseignement public, promotion d'une sécurité sociale universelle et amélioration des services publics urbains (transports en commun notamment).

Une première convergence mérite d'être soulignée: la cause des problèmes sociaux est le chômage pour les deux auteurs. Or, on oppose souvent la France et les États-Unis comme deux cas polaires : les ÉtatsUnis connaîtraient un faible taux de chômage et un taux élevé de pauvreté laborieuse tandis que la France aurait un taux de chômage élevé et une pauvreté laborieuse beaucoup moins répandue. Il peut donc paraître surprenant que Wilson fasse de la disparition de l'emploi peu qualifié la cause centrale 
des problèmes sociaux américains. Mais en vérité c'est moins la raréfaction quantitative de l'emploi que l'affaissement qualitatif de la norme d'emploi que le sociologue américain désigne comme cause. Si cette norme d'emploi s'est écroulée, c'est que le travailleur a été abandonné par la société et laissé dans un face à face perdant avec l'employeur : outre le gel du salaire minimum pendant dix ans, la faiblesse des équipements collectifs (crèches et transports en commun notamment) et des assurances sociales conduisent trop souvent les travailleurs peu qualifiés à l'impossibilité de proposer leur force de travail. Son analyse rejoint donc celle de Castel pour voir la source des difficultés actuelles dans la crise de la relation salariale telle que le fordisme l'avait normalisée au cours de la longue période de croissance de l'après-guerre.

Ce constat commun pourrait paraître banal si l'on ne soulignait pas à quel point il va à l'encontre de la « handicapologie » dans laquelle les discours politique, administratif et scientifique ont enfermé la réflexion sur les inégalités et la pauvreté depuis les années quatre-vingt. C'est la seconde convergence notable entre les deux ouvrages : ils critiquent chacun les notions qui ont colonisé les univers intellectuels et les catégories d'intervention publique, «l'exclusion» en France, «l'underclass » aux ÉtatsUnis. Sans entrer dans le détail des signifiés subsumés par chacune de ces deux notions - elles ne sont d'ailleurs pas exactement équivalentes —, on peut noter qu'elles présentent selon Castel et Wilson un tort théorique commun : elles autonomisent un ensemble disparate de situations périphériques et rendent invisibles les processus qui les produisent. Opérant une typologie des «parcours » individuels qui conduisent aux situations les plus dramatiques, elles envisagent les remèdes comme une série d'actions ciblées et non comme une autopréservation de la société. C'est cette conversion du regard que, dans des termes différents, les deux auteurs s'attachent à produire. On comprend par là que ces derniers soient également critiques vis-à-vis du discours moral sur la pauvreté. Mais leur analyse porte sur des aspects différents. Castel borne sa critique explicite du discours substantialiste chrétien sur la pauvreté et montre que ce discours est surtout propre à maintenir les pauvres assistés sous tutelle, dans le giron de la morale. Toutefois, c'est aux acteurs de l'assistance privée (chrétienne) du passé que Castel réserve ses flèches les plus acérées. Wilson condamne les tentatives contemporaines de responsabilisation des désaffiliés. Il montre avec fermeté que les comportements déviants rencontrés parmi les populations précaires ne sont pas la cause mais la conséquence de leur situation et, pour réfuter les préjugés les plus courants sur les populations démunies, il conduit une argumentation serrée utilisant le raisonnement «toutes choses égales par ailleurs ».

Il faut dire ici que la situation américaine et la situation française sont, sous ce rapport, bien différentes. D'abord, si une certaine humeur libérale a 
prévalu en France, les chiffres élevés du chômage ont sans doute beaucoup contribué à ce que ne se diffuse pas un discours rendant les pauvres coupables de leur situation. Aux États-Unis le discours - conservateur à l'origine - de responsabilisation de la pauvreté a si bien étendu son emprise qu'il a rallié les démocrates, auteurs quelques mois après la parution du livre de Wilson d'une importante réforme (réduction) du Welfare. Ensuite, l'immense majorité de la population désaffiliée aux États-Unis est africaine-américaine et le discours d'accusation morale des pauvres est lourd de préjugés racistes. Sans faire du racisme la cause première de la ségrégation - au contraire, selon lui le stigmate racial n'existe que par le stigmate de classe qu'il démultiplie —, c'est aussi à combattre les préjugés racistes que s'attache Wilson lorsqu'il réfute le discours moral sur la pauvreté. La réflexion sur l'ethnicisation des rapports de classe, réalité sans doute moins prégnante en France, est en revanche quasiment absente de l'ouvrage de Castel.

Mais, au-delà des ces différences nationales, c'est bien la même handicapologie — de l'analyse comme de l'(in)action publique — qu'entendent dépasser Castel et Wilson lorsqu'ils montrent que l'emploi ne peut être abandonné au face à face privé entre travailleurs et employeurs mais doit être inscrit dans des stratégies collectives par le seul tiers qui puisse en garantir la forme salariée : l'État.

Frédéric VIGUIER

Federico Neiburg, Los Intelectuales y la invencion del peronismo. Estudios de antropologia social e cultural. Buenos Aires, Alianza Editorial, 1998. $13 \times 20$, 290 p. [Édition brésilienne: Os Intelectuais e a invenção do peronismo. Estudos de antropologia social e cultural. São Paulo, EDUSP (Ensaios latinoamericanos), 1997].

Cet ouvrage constitue l'adaptation de la thèse de doctorat en anthropologie soutenue par l'auteur à Rio de Janeiro en 1993. Federico Neiburg (professeur et chercheur au Museu Nacional de l'université fédérale de Rio de Janeiro) ne retrace pas dans ce livre l'histoire du parti péroniste, ni le parcours du colonel Perón, ni les événements de la période qui l'a vu gouverner le pays (1943-1955). Pourtant, en analysant le très vaste débat qui, depuis la chute de Perón en 1955, a eu le péronisme comme objet, l'auteur 
nous permet de comprendre pourquoi ce phénomène représente encore aujourd'hui, pour ses adversaires autant que pour ses partisans, une catégorie fondamentale de la culture nationale, un « concentré d'argentinité ». Sa réflexion sur la relation constituante entre les « représentations de la réalité » et la «réalité », nous montre donc que les débats sur le péronisme font partie de sa propre histoire.

En prenant comme matériel ces débats, l'auteur analyse comment le péronisme est devenu après 1955 le sujet le plus légitime (il semblait nécessaire aux intellectuels argentins de comprendre l'origine et la nature de ce phénomène) et le plus légitimant (il était à l'époque indispensable, pour être reconnu comme intellectuel, de fournir une interprétation du péronisme). Il s'agit donc, pour Neiburg, de comprendre comment les intellectuels argentins de l'époque ont construit (inventé) le péronisme, mais aussi d'analyser comment cette construction leur a permis d'assumer et de légitimer leur statut d'intellectuels.

Le choix de cette problématique rend ce livre intéressant même pour des lecteurs qui ne sont pas spécialistes du péronisme ou de l'Argentine et qui s'intéressent au rôle des intellectuels dans la construction de représentations socialement signifiantes comme la nation, l'histoire, l'identité culturelle ou politique.

L'auteur a développé cette problématique en six essais relativement indépendants : les discours qui, après 1955, ont eu le péronisme comme objet; la genèse sociale des protagonistes de ce débat; les relations existant entre les interprétations du péronisme et les mythes nationaux; le rôle du Colegio de Libres Estudios Superiores, la plus importante institution antipéroniste sur le plan culturel et politique; la manière dont Gino Germani, le fondateur de la « sociologie scientifique », « invente » et légitime un point de vue spécifique, dans le débat sur le péronisme, qui est celui des sciences sociales et du sociologue; enfin, le dernier essai nous montre comment a eu lieu, après 1955, le processus de dé-péronisation dans le corps enseignant de l'université de Buenos Aires. Étant donné la problématique développée dans ce numéro, je me concentre ici sur l'analyse de l'interprétation du péronisme fournie par la sociologie et de la reconnaissance sociale et académique de cette discipline (et de ses spécialistes) que le succès de cette même interprétation a rendu possible.

Le péronisme était considéré comme un phénomène «typiquement argentin » dont la compréhension serait fondamentale. En effet, aux yeux des contemporains, comprendre le péronisme signifiait comprendre l'Argentine et ses dilemmes nationaux. Depuis la fin du $\mathrm{XIX}^{\mathrm{e}}$ siècle, l'Argentine a été considérée par les intellectuels locaux comme une nation en crise déchirée entre la civilisation et la barbarie, la modernité et la tra- 
dition, Buenos Aires et la province. Ce diagnostic constitue d'ailleurs une constante pour tous les pays latino-américains dont les intellectuels, depuis la fin du XIXe siècle, ne cessent de dénoncer le fait que leurs pays ne constituent pas de véritables nations à cause du manque d'intégration de leur population composée de groupes différents et parfois même ennemis : indigènes-européens, montagnards-habitants de la côte, campagnards-citoyens. L'auteur ne fait aucune référence à la production d'intellectuels d'autres pays, isolant le débat argentin du contexte latino-américain. Pourtant, les thèmes de la crise nationale et de la non-intégration étaient débattus au niveau du continent, certes avec des spécificités nationales, depuis la fin du $\mathrm{XIX}^{\mathrm{e}}$ siècle, par des intellectuels qui communiquaient par-delà les frontières nationales. Un aperçu, même rapide, de ce débat latino-américain sur la nation et sa crise aurait certainement enrichi la compréhension des catégories mises en œuvre par les intellectuels argentins dans l'explication du péronisme.

Le colonel Perón avait trouvé son électorat parmi les ouvriers agricoles et les prolétaires venus de la province pour chercher du travail dans l'industrie de Buenos Aires, pour lesquels il avait pris des mesures de protection sociale et d'augmentation salariale. Il a pour cela été associé à la « vraie Argentine » populaire (pour ses défenseurs) et à la barbarie (pour ses adversaires). Le péronisme a donc été considéré par ses opposants comme une nouvelle manifestation de « la crise argentine » due à l'irréductible différence existant entre deux types de populations. Par contre, pour ses partisans, le péronisme avait résolu la crise en intégrant le peuple à la nation. Le fait que penser le péronisme signifiait, aux yeux des contemporains, penser l'Argentine et sa crise, donnait au débat sur le péronisme une grande légitimité. Cette légitimité était accrue par le fait que, pour tous les interprètes de l'époque, la base sociale du péronisme avait été le peuple. Il s'agissait donc de discuter sur le pourquoi de cette adhésion populaire, mais aussi de décider ce qu'il fallait faire avec le peuple péroniste, qui en 1955, avait perdu son chef. Ainsi, chaque interprétation était également un projet de société. Chaque interprète du péronisme essayait alors de définir la place qu'il aurait occupé dans la société postpéroniste qu'il envisageait. Donc, chaque interprétation était également une définition de l'identité sociale de l'interprète. Il existait alors une véritable lutte entre les intellectuels pour s'affirmer comme les prophètes capables de révéler une des plus importantes énigmes nationales. De cette manière, la légitimité et l'importance du débat ont contribué à la construction du péronisme en tant que représentation et en tant que phénomène social encore aujourd'hui signifiant.

L'imbrication existant dans ce débat entre une description du phénomène (ce que le péronisme a été), une formulation normative (ce que la société 
postpéroniste doit être) et une production identitaire (quelle sera la place de l'interprète dans cette société) caractérise aussi les productions des spécialistes des sciences sociales. Gino Germani (1911-1979), le «père fondateur» de la «sociologie scientifique» (une sociologie basée sur des méthodes empiriques, notamment statistiques, qui s'opposait à la sociologie spéculative de l'époque), partageait le diagnostic de la crise argentine et voulait donner de celle-ci une description sociologique. Le péronisme s'expliquait pour Germani dans un contexte de changement qui avait entraîné une situation d'anomie sociale pour les migrants d'origine agricole déracinés et prolétarisés. Ces derniers cherchaient alors des repères dans des formes d'autorité paternaliste auxquelles la vie à la campagne les avait habitués et choisissaient pour cela le péronisme. Ainsi, Germani considérait le péronisme comme une survivance de la société traditionnelle (agricole, provinciale) dans la société moderne (industrielle, citadine). Le problème de l'Argentine était donc pour lui le manque d'intégration de la société : le péronisme avait été une forme perverse, puisqu'il s'agissait d'une forme autoritaire, pour le résoudre et la dépéronisation devait proposer des alternatives meilleures. En formulant les choses de cette manière, Germani créait une demande pour la sociologie scientifique et pour le sociologue, qui apparaissait comme le spécialiste capable de traiter les conséquences sociales du changement. Cette formulation sociologique impliquait aussi un projet de société : il s'agissait d'une société moderne qui puisse intégrer le peuple de manière démocratique. Mais alors, le sociologue scientifique, tel que Germani le préconise, n'avait pas seulement la capacité de théoriser la transition, mais aussi de la planifier. Il était en même temps un spécialiste et un agent de la modernisation.

La «sociologie scientifique » a été reconnue en Argentine (socialement et institutionnellement) parce qu'elle a su proposer une explication des dilemmes nationaux qui était, en même temps, une interprétation «scientifique » du péronisme. En interprétant le péronisme, la sociologie de Germani a donc pu s'affirmer comme la «voix de la science ». Parallèlement, la présence d'une interprétation reconnue comme scientifique dans le débat, a eu comme effet de sanctionner scientifiquement l'existence sociale du péronisme. Ainsi, la sociologie scientifique a participé à la légitimation du péronisme et de la dépéronisation comme problèmes.

Toutes les études sur les mythologies nationales, dont celles sur le péronisme, sont confrontées au risque de produire une nouvelle version des mythes qu'elles se proposent d'analyser. Neiburg réussit à éviter ce travers en partant du caractère construit des réalités nationales, et des mythes qui les légitiment, et en décrivant la logique sociale sous-jacente à la naissance de ces mythes et de leurs interprètes. Ayant écarté une perspective objectivante, il peut nous montrer la richesse des processus sociaux d'invention 
des « dilemmes nationaux » et surtout le rôle des sujets impliqués dans ce processus qui, en se légitimant comme les interprètes de ces dilemmes qu'ils contribuent à inventer, fondent ainsi leur position sociale.

Martina AvanZA

Antonio Carlos de Souza Lima, Um grande cerco de paz. Poder tutelar, indianidade e formação do Estado no Brasil. Petrópolis, Vozes, 1995. 13,8 × 21, 335 p., cahier iconogr., bibliogr.

Claude Lévi-Strauss raconte dans Tristes tropiques avoir pris son «premier contact avec les sauvages » du Brésil en suivant la tournée chez les Kaingang d'un fonctionnaire du Service de protection des Indiens (SPI). Il indique aussi que les Nambikwara chez qui il séjourne en 1938, longtemps hostiles, ont été «contactés » à partir de 1907 par la «commission Rondon». Ce contexte de la «rencontre ethnographique» est souvent méconnu, alors même qu'il joue un rôle essentiel dans l'histoire de l'ethnologie au Brésil. Pourtant, l'histoire du SPI était racontée à partir des témoignages de participants : sa création en 1910 était ainsi présentée comme le fruit de la généreuse intention du colonel Cândido Rondon de «protéger» les Indiens face à des projets prévoyant leur « extermination» pour laisser le champ libre aux colons. Prenant le contre-pied de cette vision héroïque, Antonio Carlos de Souza Lima a réalisé, à partir d'une vaste consultation des archives du SPI, un passionnant travail d'ethnographie de l'État.

Une première partie théorique (s'inspirant notamment de Weber et de Foucault) met en place les notions de «conquête » et de "pouvoir tutélaire » comme forme de pouvoir d'État. Une deuxième partie évoque les circonstances de la création du Service, et le projet de ses fondateurs. La troisième étudie, à partir des rapports, à la fois les techniques de pacification, le fonctionnement des postes et le processus de «définition juridique de l'Indien » (avec l'émergence d'une codification spéciale). La dernière partie évoque les «vicissitudes » de l'histoire du Service jusqu'à la suppression en 1967 du SPI, remplacé peu après par la FUNAI (Fondation nationale d'aide aux Indiens), toujours en activité aujourd'hui.

Le point de départ de Souza Lima est d'analyser la «pacification» comme une forme de "guerre », à la fois alternative et prolongement d'un mode de relation aux indigènes qui est la Conquête (et non une "découverte »). Le titre de l'ouvrage reprend la métaphore utilisée par Rondon pour désigner l'objectif du Service : réaliser un «grand encerclement de 
paix ». Ce lexique guerrier est lié au rôle essentiel joué par les militaires républicains dans le SPI. C'est en tant que chef de la Commission de lignes télégraphiques stratégiques du Mato Grosso à l'Amazone que Rondon, ingénieur militaire de formation, s'est lancé dans l'exploration de territoires inconnus du Brésil central. De façon assez logique, le SPI sera un moment rattaché au ministère de la Guerre. Il s'agit bien d'achever la « conquête » du Brésil, c'est-à-dire de conquérir des zones encore «sauvages » au sein même de l'espace national. La «pacification » des populations va de pair avec la prise de contrôle de «territoires ». Une attention particulière est portée aux communautés indigènes situées près des frontières : imposer la protection sur les Indiens, c'est aussi protéger les frontières, faire parvenir l'État national jusqu'aux limites de ce qui est défini comme son territoire. En 1923, un membre du SPI déclare à propos de la commission Rondon : «De fait, grâce à l'abnégation de ses membres, elle a déjà conquis de larges portions du territoire national, qui jusqu'alors étaient restés étrangers à notre état émergent de civilisation. Et, avec le territoire, elle a aussi fait la conquête des populations qui y étaient plus ou moins localisées. »

En rappelant que le SPI fut créé sous le nom de « Service de protection des Indiens et de localisation des travailleurs nationaux », Souza Lima met en lumière une dimension essentielle de cet organe bureaucratique, dont l'objectif explicite était de « domestiquer » les Indiens, caractérisés par leur « extériorité », afin de les transformer en «travailleurs nationaux ». En particulier, l'enjeu est de sédentariser (autour de "postes », de lieux de peuplement indigènes et de « centres agricoles ») des populations dont le mode de vie nomade exigeait un espace de parcours considérable, libérant ainsi des terres pour la colonisation ou l'exploitation des ressources naturelles, tout en fournissant de la main-d'œuvre. Cette logique aboutit à la création de « réserves indigènes », portions de terre reconnues par l'administration comme étant «d'usufruit » pour les Indiens (mais ayant le statut de «terres publiques »), et attribuées juridiquement à une population indigène particulière. Pour les positivistes (qui ont joué un rôle important dans les débuts de la République brésilienne) à l'origine de cette création, la notion de « protection fraternelle » des Indiens doit se substituer à la «catéchèse », travail de « civilisation » des Indiens auparavant confié par l'État colonial portugais, puis par l'État impérial, aux missionnaires : il s'agit d'établir un monopole étatique du contrôle sur les populations indigènes. En même temps, les créateurs du SPI revendiquent une continuité avec les techniques de pacification des Indiens « hostiles », employées par les Jésuites dès le premier siècle de colonisation du Brésil et fondées sur une combinaison de séduction et de menace. L'auteur analyse les techniques d' « attraction» et de «domestication » à partir du récit de la pacification des Parintintin, de 
1921 à 1925, par le grand ethnologue Curt Nimuendaju, qui a longtemps travaillé pour le compte du SPI (un des intérêts de l'ouvrage est le grand nombre de documents présentés).

L'action du SPI est fondée sur un postulat « anthropologique » quasiment rousseauiste : les Indiens ont été souvent corrompus par un contact avec des « civilisés » méritant à peine ce qualificatif, qui leur ont fait subir des violences ou les ont entraînés dans un processus de dégradation morale marqué par la paresse, l'alcoolisme et la prostitution. Selon un rapport de 1912 : «Plus ils sont éloignés de la civilisation, meilleurs sont les Indiens, et plus assimilables, et plus ils sont liés à elle, moins ils sont susceptibles de progrès. [...] Tout montre que s'ils sont éduqués, les Indiens sauvages doivent progresser, moralement, intellectuellement et pratiquement. » À cette conception correspond une division du travail du SPI en quatre phases qui doivent assurer l'intégration progressive des «Indiens sauvages », cible privilégiée, à la « civilisation »: « attraction d'indiens sauvages — transformation et éducation des Indiens semi-sauvages — enseignement de travaux agricoles et industriels aux Indiens semi-civilisés — établissement des Indiens dans leur émancipation et introduction définitive à la vie civilisée » (p. 136). L'objectif est de transformer ces nationaux qui s'ignorent en «Brésiliens » fiers de leur appartenance à une «communauté nationale » (sans être pour autant des « citoyens » de plein droit). Pour cela, toute une pédagogie est développée, qui passe par l'usage des symboles : carte du Brésil, hymne national et drapeau (p. 192-193).

En même temps, le SPI se pose en médiateur nécessaire entre les «Indiens » et les « nationaux ». Pour désigner cette forme de pouvoir spécifique exercée par l'État, Souza Lima utilise la notion de «pouvoir tutélaire » (poder tutelar). Le Code civil de 1917 (complété en 1928 par une loi spécifique) pose le principe de l'incapacité civile relative des Indiens (comme les prodigues, les fous, les mineurs et les femmes mariées), les plaçant ainsi sous le régime juridique de la «tutelle ». Le SPI et ses agents sont donc devenus les tuteurs de ces «mineurs », qu'ils sont chargés de représenter (notamment juridiquement).

Souza Lima montre remarquablement que la construction de catégories classificatoires (non seulement la catégorie juridique d'Indien, définie comme nécessitant la «tutelle», mais aussi la définition de «tribus » liées à des «territoires ») et la construction d'un segment de l'État (destiné à assurer cette tutelle) sont deux processus concomitants. L' «indianité » n'est donc pas une caractéristique essentielle de ceux qui sont désignés comme Indiens, mais une construction juridique et politique imposée du dehors. Ainsi, la production de discours sur les «Indiens» (y compris le discours revendiqué comme «scientifique » de l'anthropologie) doit être analysée en lien avec les pratiques dirigées vers les populations indigènes. 
On peut noter que la stabilisation de la dichotomie Indien/non-Indien a elle-même un rôle structurant pour la division du travail scientifique qui caractérise les sciences sociales au Brésil, entre les «ethnologues », qui étudient les «Indiens », et les autres «anthropologues» (ou les sociologues), qui étudient les autres groupes.

Un des grands mérites de l'ouvrage est de ne pas se contenter d'analyser les discours produits par et sur le SPI, mais aussi d'en mesurer les limites (qui, comme souvent lorsqu'il s'agit de la situation des Indiens au Brésil, ont une dimension tragique). Souza Lima montre que l'histoire heurtée du SPI est le produit des transformations politiques nationales, et surtout des luttes au sein même de l'appareil bureaucratique, entre divers réseaux. Ballotté d'un ministère à l'autre, et toujours en position dominée dans l'appareil d'État, subissant parfois des coupes drastiques dans son budget, le SPI, avec un personnel réduit, n'a en fait pas les moyens matériels de garantir la « protection » qu'il promet aux Indiens, et son action est marquée par la discontinuité, et par des compromis locaux qui contredisent ses principes. C'est l'accumulation de dénonciations d'abus qui conduira en 1967 à l'extinction du SPI.

L'ouvrage est parfois de lecture difficile, tant du fait de l'accumulation de données que du style sinueux de l'auteur, sans parler des allusions parfois cryptiques aux débats intellectuels brésiliens; on peut déplorer l'absence d'un index, qui faciliterait la consultation. Cependant, l'effort en vaut la peine. Non seulement Souza Lima apporte une contribution majeure à une sociologie historique de la construction de l'État au Brésil, notamment dans ses rapports avec les populations indigènes, et de la production de savoirs sur les indigènes, mais il ouvre des perspectives stimulantes pour d'autres recherches sur les rapports entre l'État et des populations définies comme devant être «prises en charge », que ce soit pour analyser l'action que mène aujourd'hui l'Institut national de colonisation et de réforme agraire (INCRA) auprès des bénéficiaires de parcelles, ou les pratiques de contrôle des populations et des territoires dans d'autres contextes coloniaux et postcoloniaux. Il faut espérer qu'une traduction permettra de rendre ce travail accessible aux non-lusophones!

Benoît de L'Estoile

Indigenismo e territorialização. Poderes, rotinas e saberes coloniais no Brasil contemporâneo. Dir. João PACheCo De Oliveira. Rio de Janeiro, Contra Capa, 1998. $13,5 \times 21,311$ p. (Territorios sociais).

Ce livre démontre que l'anthropologie appliquée n'est pas automatiquement condamnée à une attitude militante, mais au contraire peut être un 
outil technique extrêmement précis pour l'évaluation des pratiques politiques existantes, et de cette manière apporter des solutions scientifiques à un problème social.

Le problème abordé est la politique brésilienne relative à la question foncière des terres indigènes au long $\mathrm{du} \mathrm{xx}^{\mathrm{e}}$ siècle, riche en changements socio-politiques. La collection d'articles présentée dans ce livre est le résultat d'une recherche intitulée PETI (Projet d'étude sur les terres indigènes au Brésil : invasions, utilisation des terres et ressources naturelles), publié avec le concours de la fondation Ford. Sous l'égide de João Pacheco de Oliveira, anthropologue du Musée national (université fédérale de Rio de Janeiro), une équipe de chercheurs a mené des investigations entre 1983 et 1993. Ce projet a engendré diverses publications scientifiques, dont plusieurs thèses de maîtrise et de doctorat, traitant des problèmes liés à la question des terres indigènes comme la politique indigéniste, l'ethnogenèse et les mouvements indigènes.

Le lecteur est progressivement amené à la découverte de la complexité du problème des terres indigènes. Au premier chapitre, Pacheco de Oliveira analyse le concept de «terre indigène » tel qu'il est conçu par la loi n ${ }^{\circ} 6001$ du 10 décembre 1973, plus connue sous le nom de «statut de l'Indien ». Les implications sociologiques de cette conception relèvent de l'idéologie colonialiste et évolutionniste. Ainsi la relation entre le nombre d'indigènes par hectare est-elle pensée à partir de catégories de productivité agricole, c'est-à-dire basée sur le présupposé d'une évolution inéluctable des indigènes vers une vie paysanne, thème repris par Antonio Carlos de Souza Lima au chapitre vII. Dans ce sens, seul le principe de la propriété collective distingue encore les indigènes rassemblés dans des réserves ou parcs nationaux des paysans brésiliens - une distinction qui, dans l'esprit du législateur brésilien, devrait peu à peu disparaître. Le "statut de l'Indien » ne tient donc pas compte d'une catégorie sociologique, mais définit simplement la situation juridique des indigènes. Celle-ci est caractérisée notamment par la tutelle en lieu et place des droits civils, ce qui affaiblit davantage la combativité sociale des indigènes. L'attitude face aux indigènes est issue en droite ligne des principes coloniaux. Ce substrat donne le ton à la politique indigéniste au Brésil et est relevé dans l'analyse tout au long de la lecture.

On peut dégager une relation entre la démarcation des terres indigènes, l'ancienneté des contacts interethniques, le nombre d'indigènes présents dans une région et l'étendue des terres revendiquées. Beaucoup d'indigènes qui sont depuis longtemps, à travers les fronts pionniers, en contact avec la société brésilienne ont vu leur situation foncière réglée par le Service de protection des Indiens (SPI), l'organisme antérieur à la Fondation nationale de l'Indien (FUNAI). Mais ces réserves sont trop petites pour assurer 
leur survie, ce qui condamne les indigènes à la prolétarisation — autrement dit, ils deviennent un réservoir de main-d'œuvre agricole. La motivation politique sous-jacente est évidente, puisque le but ultime est l'intégration des indigènes à travers l'acculturation dans la société nationale. De surcroît, l'éparpillement des compétences et le manque d'un concept général et de longue durée dans la politique indigéniste ouvrent les portes à la corruption. Le «flou artistique » de cette politique permet au gouvernement de bloquer la démarcation des terres indigènes, où une exploitation à ses yeux économiquement plus rentable se présente. Un contrôle par la société civile s'impose. L'analyse objective, par des études anthropologiques, du processus administratif de la démarcation des terres indigènes semble un premier pas dans ce sens mais, pour ce faire, il faut encore assurer à l'anthropologue une meilleure position dans les rapports de pouvoir avec le champ politique.

Le deuxième chapitre approfondit la question de l'étendue des terres revendiquées et/ou contrôlées par les indigènes; dans quelle mesure cela signifie-t-il un blocage du développement agricole, comme le prétendent bien des agents sociaux au Brésil? Répondre à cette question signifie d'abord informer la société brésilienne, trop souvent malmenée par les informations contradictoires et simplistes des canaux officiels. Pas à pas, le processus administratif de l'identification des terres indigènes jusqu'à leur démarcation est étalé devant le lecteur, et le décalage visible entre ce que la FUNAI devrait faire et ce qu'elle a réalisé ne laisse aucun doute sur son inefficacité. En fait, la FUNAI se bat en premier lieu pour reproduire ses propres structures; de ce fait, la démarcation des terres indigènes est ralentie, ce qui permet entre-temps une invasion progressive des terres indigènes qui complique encore davantage la situation : un cercle vicieux s'installe.

La critique de la classe économique brésilienne à la fin des années soixante-dix à propos de la politique indigéniste soulignait toujours l'absurdité de la relation entre le petit nombre d'indigènes et les grandes aires qui leur sont réservées. Le blocage du développement du Brésil en a été vite déduit. La question foncière devint alors une question de sécurité nationale, ce qui compliqua d'avantage la procédure de démarcation. Une analyse de la répartition des indigènes par municipalité et par microrégion montre par contre à quel point un recadrement des dimensions peut changer la perception de la situation : alors que dans les statistiques globales les indigènes ne représentent que $0,16 \%$ de la population totale, des statistiques par région montrent qu'au Roraima, par exemple, les indigènes représentent $72 \%$ de la population rurale. Leur présence est significative dans beaucoup de municipalités en Amazonie, et souvent ils y représentent la majorité. La relation de force politique prend tout de suite une autre allure.

La distorsion des revendications indigènes sert entre autres à masquer la prédominance des grands propriétaires au Brésil, dont les terres ne sont pas 
plus productives que les terres indigènes. Aussi existe-t-il encore suffisamment de terres disponibles pour une expansion de l'agriculture. 184,951 millions d'hectares sont inexploités au Brésil, dont les terres indigènes ne représentent que $40 \%$. Prendre l'indigène comme bouc émissaire d'une éventuelle stagnation du développement agricole revient à dévier l'attention d'autres facteurs beaucoup plus cruciaux et à surestimer l'impact des indigènes sur l'économie nationale brésilienne.

Quand on regarde le fonctionnement de la FUNAI tel que décrit au troisième chapitre par João Pacheco de Oliveira et Berno de Almeida, on comprend mieux son inefficacité. Une organisation qui tourne dans une logique d'urgence et où le cadre de fonctionnaires se constitue sans se soucier d'homogénéiser leurs qualifications, notamment celles des fonctionnaires qui effectueront l'expertise anthropologique, se disqualifie ellemême. C'est ainsi que la description de ses procédures ressemble plutôt à la reconstitution d'un crime. En excluant d'emblée les indigènes de la procédure comme partenaires à part entière et en reléguant les anthropologues à un rôle secondaire, l'identification des terres indigènes se déroule selon un modèle colonial qui cherche à perpétuer le statu quo.

Linhares explicite ce dysfonctionnement généralisé par la coopération institutionnelle entre la FUNAI et l'INCRA, l'organisme qui régularise l'attribution des terres publiques, dont les terres indigènes. Les tergiversations de la FUNAI font que l'INCRA finit par attribuer beaucoup de terres indigènes à des particuliers. En d'autres termes, la FUNAI travaille aux dépens de ceux qu'elle est censée protéger. Lobato nous fournit un autre exemple concret, un conflit frontalier entre les Potiguara de l'État de la Bahia et un propriétaire. La FUNAI se montre impuissante face aux préjugés racistes de l'appareil juridique chargé de traiter l'affaire, et ne réussit pas à faire valoir le savoir indigène concernant l'étendue de leur territoire.

À la lumière de la procédure d'identification des terres indigènes, effectuée institutionnellement par un groupe de travail qui réunit diverses compétences, dont celle de l'anthropologue, Souza Lima montre à quel point le substrat colonial et évolutionniste s'est installé dans la façon de penser le problème indigène. L'anthropologue se trouve dans le rôle de l'avocat du diable : il est obligé de trouver les éléments historiques pour la légitimation des demandes foncières indigènes dans une situation où le savoir indigène est démuni de reconnaissance sociale, alors qu'il constitue pratiquement la seule source d'information pour l'anthropologue. Cela est approfondi au chapitre suivant, quand l'analyse des rapports met en évidence l'absurdité d'une bureaucratie qui normalise les démarches administratives afin de se créer une image objective, voire scientifique, mais en reniant son rôle éminemment politique. Le mimétisme avec le monde académique produit un discours métissé dans lequel les pratiques coloniales se 
perpétuent en toute discrétion. Pacheco de Oliveira insiste dans le dernier chapitre sur la difficulté de combiner le savoir et le savoir-faire anthropologiques avec la logique administrative lors des expertises anthropologiques pour la FUNAI.

Ce livre est un plaidoyer pour l'étude anthropologique des systèmes administratifs. Il souligne l'incompatibilité de la dynamique des processus sociologiques avec les procédures administratives qui n'en tiennent pas compte. La complexité des thèmes liés à ce genre d'analyse, allant de la formation scolaire et universitaire à l'épistémologie, nous rappelle que chaque société est un fait social qui doit être étudié dans son ensemble. Cette radiographie de la politique indigéniste brésilienne ne dénonce pas simplement les pratiques coloniales, mais propose des solutions concrètes et ancrées dans une analyse objective. L'anthropologie politique a bel et bien une application, sans tomber dans le militantisme.

Un seul regret toutefois suite à la lecture de cet excellent ouvrage : un chapitre supplémentaire aurait été le bienvenu à titre de contrepoids anthropologique rigoureux à la façon administrative d'établir le territoire et l'ethnicité des indigènes. Le lecteur pourrait ainsi apprécier pleinement l'anthropologie comme science au service de la société. Un deuxième volume nous donnera peut-être ces informations et présentera l'évolution des événements de la politique indigéniste à partir de 1994. À espérer.

Tomke LASK

Anthropologues en dangers. L'engagement sur le terrain. Éd. Michel AgIER. Paris, Jean-Michel Place, 1997. $16 \times 22,4,128$ p. (Les Cahiers de Gradhiva, 30).

Tout en regrettant le titre inexact et légèrement racoleur de cet ouvrage — où il n'est nullement question des risques physiques qu'il arrive parfois aux ethnographes d'encourir — on doit se féliciter de la publication de l'ensemble des réflexions rassemblées ici, qui portent plutôt sur l'inconfort moral et politique des ethnologues dans de nouveaux contextes d'enquête, et sur la remise en cause de l'anthropologie classique que cette situation implique. Elles s'inscrivent dans une série de travaux réflexifs récents, largement stimulés par le débat qui s'est développé notamment aux ÉtatsUnis sur les conditions d'une anthropologie post-coloniale (et/ou postmoderne).

À travers un ensemble d'études de cas brèves mais stimulantes, les auteurs cherchent à approfondir les notions d'engagement et d'implication dans de nouveaux contextes d'enquête. Parler de «nouveaux contextes », 
de «nouveaux objets » ou de «nouveaux engagements » suppose de préciser la nature de cette nouveauté (qu'illustre la photographie de couverture sur laquelle on voit deux vidéastes amérindiens portant leurs parures « traditionnelles » aux côtés d'un cameraman, d'un réalisateur et d'un anthropologue occidentaux rassemblés par une manifestation de protestation contre la réalisation d'un barrage hydroélectrique en Amazonie). Ici, il s'agit sans doute moins de ruptures franches que d'une série d'inflexions convergentes qui rendent caduc le modèle malinowskien de l'enquête de terrain: l'exceptionnalité décroissante de la situation de l'ethnographe face au développement du tourisme; l'émergence d'une demande sociale et d'un contrôle local de l'anthropologie, pouvant confiner à la censure; des demandes (étatiques ou communautaires) d'intervention ethnologique sur le mode de l'expertise, et qui prennent souvent la forme d'enquêtes rapides. Signalons au passage que cet ouvrage est issu d'un atelier de recherche tenu à l'ORSTOM (devenu depuis l'Institut de recherche sur le développement), organisme au sein duquel les anthropologues sont plus que dans les autres instituts publics de recherche français conduits à mener des travaux d'anthropologie appliquée ou à jouer un rôle d'experts. L'éditeur et introducteur de ce recueil, Michel Agier, invite à tenir compte de la forme actuelle des terrains anthropologiques pour y abandonner trois attitudes: «l'élitisme » du début du siècle, ancré dans une relation inégalitaire entre l'ethnographe et ses interlocuteurs, et dans la sélection d'informateurs érudits; le «populisme » militant de l'après Seconde Guerre mondiale et des années soixante, critique de la colonisation et découvreur du «peuple»; et enfin le «corporatisme » actuel de la profession, repliée sur l'autoaffirmation de son statut scientifique. Agier invite donc les ethnologues à tenir compte des conditions réelles de production de leur savoir afin d'inscrire la relation ethnographique sous le signe d'un «engagement raisonné », qu'illustrent les textes rassemblés dans ce volume.

Dans la première partie, intitulée « Nouveaux contextes », trois exemples permettent d'examiner l'influence sur la recherche anthropologique, ses problématiques et ses inflexions, de ces nouveaux contextes qui installent l'ethnologue bien loin du vert paradis des tribus primitives : le développement du nationalisme hindou en Inde (Denis Vidal), la guérilla au Guatemala (Charles-Édouard de Suremain), les lendemains des guerres d'Indochine et du Vietnam au Laos (Yves Goudineau). Les auteurs montrent ainsi comment des événements inattendus ou des circonstances historiques à portée régionale, nationale ou internationale les ont amenés à reformuler, ou au moins à infléchir, leurs problématiques initiales. On s'étonne cependant que ces réflexions subtiles conduisent leurs auteurs à des positions aussi réductrices quant à la définition de l'anthropologie : Vidal l'oppose, en effet, à la sociologie (p. 38), supposée définir ses objets à partir des 
préoccupations centrales des membres de la société étudiée (alors même que c'est bien en sociologie qu'a été thématisée l'idée d'une " construction de l'objet», et que les deux disciplines se trouvent rapprochées par les «nouveaux contextes» dont il est question ici); Goudineau reproduit la posture de l'ethnologue éclairé affrontant «l'hostilité des autorités»et «l'incompréhension des villageois» (p. 62); et Suremain reconduit enfin la discipline dans sa visée structurelle, malgré la place qu'il concède aux «données immédiates », c'est-à-dire événementielles. La définition de ce qui importe est alors laissée au seul flair scientifique de l'anthropologue : «L'ethnologue qui, en prenant le temps de l'enquête longue, connaît bien son terrain n'est-il pas le mieux à même de faire la part entre ce qui ressort de l'anecdotique et ce qui relève du changement social? » (p. 49) - ce qui me semble relever de ce qu'Agier dénonce comme « corporatisme ».

L'objet de la seconde partie (« Nouveaux engagements ») est sensiblement plus intéressant : il s'agit véritablement de s'interroger sur l'implication politique, pratique et morale de l'ethnographe, c'est-à-dire sur les conditions concrètes de production du savoir anthropologique. C'est ici que s'esquisse une analyse tout à fait stimulante des rapports entre l'anthropologie, l'État et les sociétés étudiées. Les deux textes de Bernard Moizo et plus encore de Bruce Albert sont particulièrement riches en ce qu'ils thématisent l'idée d'un bouleversement des conditions de l'observation participante. Ce qui définit les conditions actuelles de l'enquête ethnographique n'est pas tant pour eux la disparition des sociétés traditionnelles ou la fermeture de l'accès au terrain que la nécessaire négociation du statut de l'enquêteur - la participation (l'apport éventuel de l'ethnographe dans les revendications identitaires ou politiques de ceux qu'il étudie) devenant la condition préalable à tout recueil de données de type ethnographique, dans une conjoncture culturaliste de revendications minoritaires face à l'État (en Australie et au Brésil, pour ce qui est des deux articles évoqués). Il y a là une place négociée à trouver, l'implication de l'ethnographe ne conduisant cependant pas nécessairement à l'instrumentalisation pure et simple de son savoir. Quand c'est cependant le cas, comme cela peut arriver en Australie aborigène (où l'accès à l'information est conditionné par les revendications foncières ou plus généralement politiques des Aborigènes), l'anthropologue se trouve réduit à louvoyer entre l'acceptation de cette instrumentalisation de ses travaux et la "fiction » (menée sous la forme d'une ethno-histoire déconnectée du présent, ou d'un anonymat généralisé portant sur les lieux et les personnes, et passant par l'évitement de dimensions de la société problématiques pour les acteurs eux-mêmes). Les deux textes suivants (de Marc-Éric Gruénais et Laurent Vidal) ainsi que la conclusion générale de Jean-Pierre Dozon s'intéressent aux problèmes éthiques liés à 
l'étude anthropologique du sida en Afrique, qui obligent les ethnographes à s'interroger sur les questions déontologiques de l'anonymat, de la neutralité de l'enquêteur, et de son rapport à d'autres types d'experts (ici, les médecins).

Cet ouvrage diversifié et stimulant peut intéresser tout à la fois ceux qui réfléchissent aux conditions de scientificité ou de rigueur des sciences sociales, à partir de l'analyse de leurs opérations élémentaires (ici l'enquête de terrain); et ceux qui cherchent à analyser le rapport entre l'État, les populations qu'il englobe, et les sciences sociales qui les étudient. Il me semble que l'analyse de la tension entre négociation de la place de l'enquêteur et instrumentalisation du savoir qu'il produit est l'apport le plus marquant du livre. On peut cependant regretter que l'étude de l'impact des changements des contextes d'enquête sur les problématiques de l'anthropologie ne s'appuie pas sur une analyse plus approfondie des catégories d'événement et de contexte, qui reste à faire.

Michel NAEPELS

Ernest Gellner, Language and solitude. Wittgenstein, Malinowski and the Habsburg dilemma. Préf. de David N. Gellner, av.-pr. de Steven Lukes. Cambridge, UK, Cambridge University Press, 1998. $15 \times 22,7$, xx-209 p., bibliogr., index.

Cet ouvrage posthume d'Ernest Gellner est issu d'un manuscrit qui, au moment de son décès, n'était pas prêt pour la publication. Repris par son fils, réorganisé et complété par lui, il apporte sa contribution à l'histoire de l'anthropologie et à l'analyse des relations entre méthodes anthropologiques et contextes nationaux. Sans pour autant adopter un point de vue déterministe, il inscrit le développement de la pensée de Wittgenstein et de l'anthropologie de Malinowski dans l'histoire intellectuelle de l'Empire austro-hongrois. La personnalité et l'œuvre du philosophe comme celles de l'anthropologue sont envisagées à partir d'une opposition radicale entre deux pensées qui constituent autant une théorie de la connaissance qu'une vision de la société et de l'existence humaine : d'une part, celle qu'il qualifie d'individualiste et d'atomiste et d'autre part, celle décrite par les termes d'organique et de romantique. Du point de vue social, l'opposition se traduit par le fossé entre les défenseurs de la communauté, les partisans de la Gemeinschaft, et ceux de la société, de la Gesellschaft. Du point de vue de la connaissance, l'individualisme atomiste conçoit le savoir comme le fruit 
d'une activité individuelle et isolée et le définit comme une libre combinaison d'idées tandis que le romantisme organique voit dans le savant l'agent d'une communauté culturelle et linguistique et insiste sur les relations entre les idées et la totalité qu'elles forment. Chronologiquement et logiquement, le romantisme organique se développe après l'individualisme atomiste : celui-ci est formulé avant même d'être expérimenté, alors que le premier se vit sans se réfléchir et ne trouve une formulation qu'en défense et critique de l'individualisme. Dans l'Empire austro-hongrois, les deux visions naissent d'abord à partir du contexte politique. Contre les libéraux cosmopolites qui défendent l'idée d'un Empire ouvert et universaliste, se développent des forces centrifuges, nationalistes, qui mettent en avant la tradition et la culture de chaque peuple au sein de l'Empire. Aux yeux de Gellner, cette émergence double et antagoniste est paradoxalement le fruit d'un même mouvement historique - l'industrialisation de la société européenne - et de l'incapacité de l'Empire à satisfaire les revendications ethniques. C'est à ces revendications fondées sur l'idée de la Gemeinschaft que s'est greffé pour la première fois dans l'Empire austro-hongrois le mouvement des études ethnographiques sur la paysannerie, destinées à collecter et à conserver les traditions de cette dernière.

Une fois mise en place cette opposition entre individualisme atomiste et romantisme organique, Gellner passe à l'évaluation des œuvres de Wittgenstein et de Malinowski dans l'histoire politique de l'Empire austro-hongrois. Celle du premier est jugée très négativement. Wittgenstein aurait, dans le Tractacus logico-philosophicus (1922), puis dans Philosophical investigations (1953), conçu une théorie de la connaissance et du langage d'abord inspirée de l'individualisme atomiste et ensuite du romantisme organique. Dans la première phase, il aurait exposé dans un style dogmatique une conception du monde comme somme de «faits » homogènes, sans envisager leur structure ni leurs relations, ainsi qu'une ontologie prétendument définitive. À partir de cette ontologie, Wittgenstein serait incapable de rendre compte de l'hétérogénéité des faits et de penser que le monde pourrait être autre qu'il n'est, autrement dit, qu'il y a une pluralité d'interprétations du monde. Enfin, le langage d'après Wittgenstein, déduit de la logique conçue au début du siècle, ne ferait aucune place au fait que nous appartenons, par notre langue, à une communauté. En bref, Wittgenstein "première manière» se présente comme un homme seul et aveugle à l'existence de la culture et de sa pluralité. Il renverrait de manière caricaturale à l'individualisme atomiste, auquel il serait, en raison de son origine sociale - une famille juive fortunée de Vienne - enclin à se rattacher: ceux qui sont venus gonfler les rangs des libéraux cosmopolites étaient les parias sociaux, et parmi eux, tout spécialement les juifs, qui avaient tout à craindre des replis communautaires et, à l'inverse, beau- 
coup à gagner des opportunités économiques liées à l'industrialisation, de la valorisation de l'individu et d'une société où la reconnaissance sociale ne reposerait pas sur l'appartenance ethnique. Cependant, le « second Wittgenstein » envisage la pensée humaine dans sa relation à une communauté culturelle spécifique et le rapport du langage à ses usages au sein des communautés, dont les visions du monde s'autovalident. En cela, Gellner voit l'exploration de l'autre voie suggérée par le contexte historico-politique de l'Empire austro-hongrois, celle du romantisme organique diffusée par les doctrines nationales-populistes. Elle serait empruntée par Wittgenstein en raison du caractère insupportable de la solitude découlant du Tractacus et de la nécessité personnelle d'y mettre fin.

Malinowski fait l'objet d'un jugement tout à fait différent. Rappelant qu'il est issu d'une famille de la haute bourgeoisie polonaise et, partant, qu'il est doté, à l'inverse de Wittgenstein, d'une identité non problématique au sein de l'Empire, Gellner souligne son rôle révolutionnaire pour l'anthropologie. En tant que fondateur de l'anthropologie sociale britannique, il a rompu avec une tradition inaugurée par James Frazer, celle d'une anthropologie évolutionniste, censée rendre compte des étapes du progrès intellectuel de l'humanité, en étudiant chez les peuples primitifs ce qui constitue la phase initiale du développement de l'esprit. Malinowski entreprend pour sa part de rendre compte du présent en établissant les liens entre les croyances observées et la société où se déroule le terrain, sa culture et les « besoins humains » qui s'y manifestent. En cela, il se montre radicalement empiriste et s'inspire de l'ethnographie pratiquée en Europe centrale. Celle-ci, dans une finalité politique, s'est attachée à protéger les cultures des peuples de l'Empire, en codifiant leurs langues, en collectant leurs coutumes, leurs proverbes, leurs contes et leurs chants. Elle a aussi cherché à comprendre chaque culture comme un tout («a unity») et à rendre compte des différences et des nuances entre chacune. Dans cette perspective, le terrain et sa durée acquièrent une importance décisive et l'on ne peut s'étonner que Malinowski considère le terrain long, l'apprentissage de la langue et l'immersion complète dans la vie des indigènes comme la condition de validité du discours anthropologique alors que Frazer utilisait des informations déjà collectées par d'autres. L'influence du romantisme organique a ainsi conduit Malinowski, selon Gellner, à transposer l'amour des cultures nationales dans le cadre d'une microsociologie des peuples colonisés.

La conclusion de Gellner est sans ambiguïté : l'œuvre de Malinowski représente une combinaison beaucoup plus réussie que celle de Wittgenstein des courants de pensée qui s'opposaient dans l'Empire austrohongrois. Il a en effet associé, à une remarquable indépendance vis-à-vis du nationalisme, une grande attention pour la culture. Il a été en ce sens à la 
fois libéral et attaché aux cultures et à leurs unités respectives. Il a en outre formulé une conception du langage en relation avec ses usages au sein d'un contexte culturel spécifique dont on attribue à tort la paternité au Wittgenstein des Philosophical investigations. Malinowski l'a développée à l'époque même où le philosophe écrivait le Tractacus.

La lecture de cette œuvre posthume exige une certaine abnégation. Il faut passer outre les interprétations superficielles que Gellner propose de plusieurs philosophes, Hume, Kant, Descartes, et sur la vision partiale de l'enquête philosophique menée par Wittgenstein. Il faut également négliger l'aspect caricatural de l'opposition entre individualisme atomiste et romantisme organique, et cela n'est pas sans difficulté dans la mesure où elle occupe une place centrale dans l'économie de l'ouvrage. On peut néanmoins en tirer un double profit. Tout d'abord, on pourra le considérer d'un œil plus indulgent si on le conçoit autant comme une autobiographie intellectuelle que comme un commentaire des œuvres de Wittgenstein et de Malinowski. De fait, Gellner évoque des thèmes qui lui sont chers et sont présents dans ses publications antérieures : la pensée de Wittgenstein, l'histoire de l'anthropologie sociale, la réflexion sur le nationalisme et la modernité. Jusque dans une certaine mesure, Gellner s'identifie à Malinowski. Il partage avec lui son attitude à l'égard du nationalisme et loue sa vision des contextes multiethniques fondée sur une reconnaissance de la culture. Toutefois, il s'en distingue en rejetant l'idée, formulée par Malinowski dans Coral gardens and their magic (1935) selon laquelle il n'existe qu'une différence de degré entre le langage des «sauvages » et le langage scientifique. Cette «erreur de Malinowski» révèle ici plus de choses sur Gellner que sur l'anthropologue originaire de Cracovie : tout en considérant que la société moderne n'a pu choisir entre individualisme atomiste et romantisme organique, il défend le premier dans le cadre d'une théorie de la connaissance. Contre le relativisme culturel, il faut selon lui préserver l'idée d'un langage qui puisse dépasser les visions du monde issues des cultures particulières. Gellner, à travers la défense d'une vérité qui transcende les cultures («culture transcending truth»), fait ainsi son autoportrait intellectuel, qu'une bibliographie de ses articles sur Wittgenstein, Malinowski et le nationalisme vient utilement compléter pour l'historien de l'anthropologie. D'autre part, sa réflexion sur le milieu dans lequel Malinowski a grandi invite à entreprendre une recherche plus approfondie sur la pratique ethnographique en Europe centrale ainsi que sur les conditions et les effets de sa transposition dans un contexte historique et politique tout à fait différent de celui de l'Empire austro-hongrois, celui de l'Empire colonial dominé par l'idée de l'Indirect Rule. La révolution malinowskienne ne consiste pas seulement à donner un nouveau statut au ter- 
rain et à rompre avec la perspective évolutionniste : elle tient aussi à une manière d'envisager les cultures des colonisés sur laquelle, à raison, Gellner attire l'attention de l'anthropologue.

Marie GaILle-NiKodimov

\section{POUVOIRS EN EXERCICE}

Georges Faraklas, Machiavel. Le pouvoir du prince. Paris, Presses universitaires de France, 1997. 11,5 × 17,5, 128 p. (Philosophies, 78).

L'analyse de Georges Faraklas propose une reconstruction de la théorie du pouvoir politique de Machiavel, qui traduit une volonté de justice sociale, dans la mesure où on établit qu'il y va de l'intérêt pragmatique du pouvoir de s'appuyer sur le peuple.

Une bonne désunion, la division sociale, qui met en présence les grands, le peuple et l'individu au pouvoir, le prince, se substitue grâce aux institutions à la mauvaise désunion, celle des factions. Le peuple y représente l'universel, et les grands ( $i$ pochi), les particuliers. Pour Machiavel, la richesse de ces particuliers est la ruine de l'État. Pour corriger l'inégalité qui en découle, un individu singulier est nécessaire, le prince, qui devra régler le rapport de l'universel et des particuliers, en faveur du peuple.

Il est de l'intérêt du prince d'opter pour le peuple, parce que ce dernier est le seul bon fondement de son pouvoir. Son rôle étant de fonder et de décider, Machiavel pose que la résolution est une force : il faut toujours se décider à décider, ne pas rester dans l'hésitation; et que l'unicité du pouvoir en est une autre. On remarquera qu'il faut être un pour décider, ce qui montre que ces deux forces sont formelles, indépendantes de leur contenu, mais interdépendantes.

La fondation d'un État est une décision qui fonde sa propre nécessité : le prince institue, et le peuple maintient. Le problème est de savoir si l'on peut fonder moralement l'État. Machiavel répond par la négative : il n'y a pas de loyauté avant la loi. Le paradoxe fondamental du politique veut qu'un tyran soit nécessaire pour faire échec à la tyrannie, et pose en même temps la nécessité d'un égoïsme maximal pour asseoir le bien commun.

La vérité du fondateur est donc décisionnelle : on peut dire qu'elle est performative, car la décision est elle-même ce à quoi l'intellect est adéquat. 